\title{
Finger Vein Recognition Using 2DGabor Combined With OECA
}

\author{
Guangdong Liu ${ }^{\mathrm{a}}$, Xiaohui Qiub \\ School of Communication \&Information Engineering, Nanjing University of Post and \\ Telecommunications, Nanjing 210003 China
}

a13512233595@163.com, bqiuxh@njupt.edu.cn

\begin{abstract}
Keywords: Optimized KECA, Optimized ECA, Feature Extraction, 2DGabor Feature Fusion, and Independent Component Analysis.
\end{abstract}

\begin{abstract}
An improved Entropy Component Ananysis algorithm is proposed in this paper,which named as Optimized Entropy Component Analysis. The OECA algoptithm which is based on the Optimized Kernel Entropy Component Analysis algorithm develops a different data transformation method. The data transformation reveals structure related to the Renyi entropy of the input space data set. Unlike the Principal Component Analysis (PCA) algorithm, the method does not necessarily use the top eigenvalues and eigenvectors of data correlation matrix. OECA takes the cumulative entropy contribution as the standard of feature extraction, then uses the Independent Component Analysis (ICA) framework to maximize the independence between the components. Applying the OECA algorithm to finger vein recognition. In the meantime, a new finger vein extraction algorithm, GOECA, is proposed in combination with 2DGabor algorithm: when the feature obtained with 2DGabor wavelet transform, five different feature fusion methods are applied then the OECA algorithm is used to reduce dimensions. The results of the experiment show the efficiency of the proposed method.
\end{abstract}

\section{Introduction}

Biometric identification is a kind of recognition technology, which usually refers to a process by which a person can be identified by human biometric information. Various recognition technologies have been developed in the past several decades, such as fingerprint recognition, iris recognition, face recognition, signature recognition, gait recognition and so on. Among all types of recognition technologies, fingerprint recognition technology is most widely used in daily life. While, the fingerprints are easily stolen and wear, so it is insufficient in terms of stability and safety. The same goes with the signature recognition. The scanning device of iris recognition integration is much more complexity than the others. Face recognition is susceptible to light condition, facial makeup. Gait recognition can be easily affected by the health and clothing wear of the observed walkers.

In contrast, as a new biometric technique, finger vein recognition uses the vein map inside the finger to identify a person, which has many unique advantages over traditional technologies: finger vein map meets the requirements of specificity and uniqueness in pattern analysis: as the age changes, finger vein structure will not change dramatically; existing inside the skin, so it can not be wear easily and stolen easily. With the development of the Internet and information processing technology, people realize that traditional biometric technology can not meet the needs of information security, Owing to the above, finger vein recognition attracts more researchers to work on this field. The earliest research literature on finger vein recognition is published by Japanese scholar Kono [1], Hatichi develop a patent finger vein recognition system [2] in 2005, since then finger vein recognition technology is applied to a vast of fields: ATM machine, car locking system, access control system and many other applications. This breakthrough makes finger vein recognition technology research received widely concerned [3].

\section{Related Work}

Literature [4] theoretically demonstrate the characteristics of finger vein as a basis of recognition. In recent years, people have done lots of work on finger vein recognition. The extracted finger vein 
feature can be divided into four classes: the contour points feature, the venous lines feature, the texture feature and the feature obtained by machine learning methods. The contour point feature is the earliest applied to finger vein recognition to extract the bifurcations point and the endpoints as the classification information [5], and then the SIFT (Scale-invariant feature transform) feature points are introduced to enrich the robustness against rotation [6], but the contour point feature will lose a lot of information. Finger vein line can reflect the vein topology, linear tracking method [7], regional growth method [8], curvature method [9] is often used to extract the vein line. LBP (Local Binary Pattern) is widely used in finger vein recognition as a class of methods with strong texture description ability [10], but at the same time it is insensitive to the changes in light intensity, Gabor filter banks can extract the global and local information of the veins, and the obtained texture features have good texture description ability and anti-noise ability [11]. The machine learning methods are also widely used in finger vein recognition. PCA can extract feature and reduce feature dimension, which has many excellent improvement methods [12], the KECA and Optimized KECA are proposed by Professor Jessen, Robert. KECA algorithm is widely used in pattern recognition [13] [14]. Optimized KECA is an extension algorithm of KECA, which can directly extract the optimal features retaining most of the data entropy by means of compacting the information in very few features. According to the Optimized KECA algorithm, we proposed the Optimized ECA, which can reduce the computation complexity. Then the fusion method of 2DGabor algorithm and OECA are proposed and apply it on the finger vein recognition.

\section{Proposed Method}

\subsection{Gabor wavelet}

Gabor filter bank is characterized as localized, frequency selective and orientation selective, which is similar to mammal's receptive field profiles in cortical simple cells. 2DGabor filter bank has been found to be particularly suitable for texture representation and object recognition, so the 2DGabor filter bank can achieve good results in image texture feature extraction.2DGabor wavelet transform is a powerful tool for multi-scale representation and analysis of images. As the only Gabor function which can obtain the lower bound of time domain and frequency domain joint uncertainty, it is often used as wavelet basis function to analyze the image. It can be formulated as:

$$
\begin{gathered}
\varphi_{u, v}(z)=\frac{\left\|\mathrm{k}_{u, v}\right\|^{2}}{\sigma^{2}} \exp \left(-\frac{\left\|\mathrm{k}_{u, v}\right\|^{2}}{2 \sigma^{2}}\right)\left[\exp \left(\mathrm{i} k_{u, v} z\right)-\exp \left(-\frac{\sigma^{2}}{2}\right)\right] \\
k_{u, v}=k_{v} \exp \left(i \varphi_{u}\right)
\end{gathered}
$$

where $k_{u, v}$ is the 2DGabor wavelet vector, $\mathrm{u}, \mathrm{v}$ define the orientation and scale of the 2DGabor kernel respectively, usually set $\mathrm{u}=5$ and $\mathrm{v}=8 . k_{v}=k_{\max } / f^{v}, \varphi_{u}=\pi u / 8, \varphi_{u} \in[0, \pi), k_{\max }$ is the maximum frequency and $\mathrm{f}$ is the kernel distance factor in frequency domain. $\sigma$ is the variance related to the wavelet frequency bandwidth, $Z=Z(x, y)$.In the experiment, we set the parameters as follow: $k_{\max }=\pi / 2, \mathrm{f}=\sqrt{2}, \sigma=2 \pi$.

According to Euler formula, Gabor filter can be decomposed into a real part and an imaginary part. The real part, usually called even-symmetric Gabor filter, is suitable for ridge detection in an image. While the imaginary part, usually called odd-symmetric Gabor filter, is beneficial to edge detection. Since the finger veins appear dark ridges in image plane, even symmetric Gabor filter here is used to exploit the underlying features from the finger-vein map.

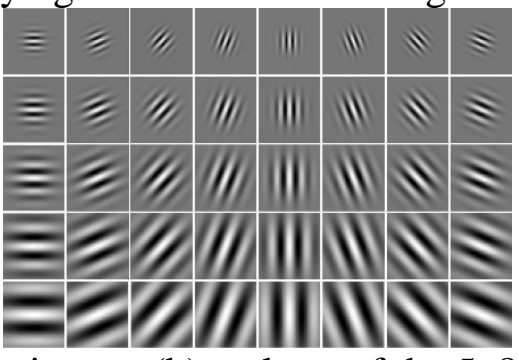

(a)finger vein image (b) realpart of the 5x8 2DGabor filter

Figure 1 . The sample of finger vein picture and the 2DGabor convolution results

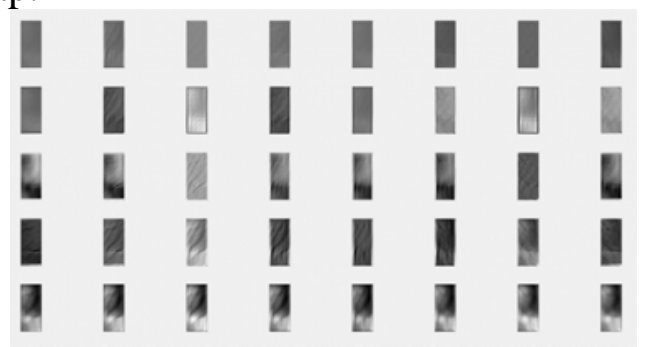

(c) the convolution results

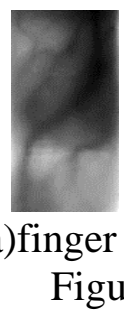


Given a finger vein image, it's 2DGabor filter transformation can be computed by a convolution with the 2DGabor kernel:

The results show in above figure1-(c).

$$
G_{u, v}(z)=\mathrm{I}(\mathrm{z}) * \varphi_{u, v}(z)
$$

\subsection{Optimized ECA}

Optimized KECA is a method of dimension reduction. Different from the PCA and KPCA algorithm, Optimized KECA algorithm do not chose the top eigenvectors of the input data sets. The Optimized KECA algorithm transfers the data from higher dimension to lower dimension are achieved in a way which contributes to the entropy estimate from input data set. Then the framework of Independent Component Analysis (ICA) algorithm is introduced to optimize the input data matrix decomposition [16]. Based on the Optimized KECA algorithm, we propose the Optimized ECA algorithm, which can reserve the advantage the Optimized KECA achieved and reduce the computation complexity. The following describe the Optimized ECA algorithm:

According to paper [15][16], we can extract the Renyi second-order entropy input data set, the formulation can be expressed by:

$$
H(p)=-\log \int p^{2}(s) d s
$$

As $H(p)$ is a monotonous function. Since we intend to order the eigenvalues according to the top second-order Renyi statistical properties, so we can use $V(p)$ instead. $V(p)$ is called "information potential” in paper [17], $V(p)=\int p(s)^{2} d s . V(p)$ can also be formulated as $V(p)=\varepsilon_{p}(\mathrm{p})$, where $\varepsilon_{p}(\mathrm{p})$ denotes expectation and $p(s)$ is the density of the input data set $S=\left[s_{1}, s_{2}, \ldots, s_{n}\right]$. We use the Parzen window to estimate the $p(s)$.

$$
\widehat{p(s)}=\frac{1}{N} \sum_{s t \in D} k_{\delta}\left(s, s_{t}\right)
$$

where, $k_{\delta}\left(s, s_{t}\right)$ is the so-called Parzen Window. so,

$$
\mathrm{V}(\mathrm{p})=\int p(s)^{2} d s=E_{p}\{p(s)\}=\frac{1}{N} \sum_{s_{t} \in D} \widehat{p\left(s_{t}\right)}=\frac{1}{N} \sum_{s_{t} \in D} \frac{1}{N} \sum_{s_{t \prime} \in D} k_{\delta}\left(s, s_{t^{\prime}}\right)=\frac{1}{N^{2}} 1^{T} K 1
$$

where $\mathrm{K}$ is a $\mathrm{N}^{*} \mathrm{~N}$ dimension matrix, $K_{i j}=K_{\delta}\left(s_{i}, s_{j}\right), 1$ is a column vector all the elements equal one, then eigen-decompose the $\mathrm{K}$ matrix [18],

$$
K=E D E^{T}=A A^{T}=\left(E D^{\frac{1}{2}}\right)\left(D^{\frac{1}{2}} E^{T}\right)
$$

where $\mathrm{D}$ is a diagonal matrix that contains the eigenvalues and $\mathrm{E}$ contains the eigenvectors. Then $V(p)$ can be rewritten as

$$
\widehat{V(p)}=\frac{1}{N^{2}} \sum_{i=0}^{N}\left[\sqrt{\tilde{\lambda}_{i}} e_{i}^{T} 1\right]^{2}
$$

where $\lambda_{i}$ and $e_{i}$ are the eigenvalues and eigenvectors of the matrix K. Since the covariance matrix is a semi-definite matrix, we can directly eigen-decompose it. The projection of Optimized ECA onto the kth principal axis in the feature space is defined as

$$
\Phi_{\text {eca }}=D_{k}^{\frac{1}{2}} E_{k}^{T}
$$

where $D_{k}$ consists of the top k eigenvalues of $\mathrm{K}$ and $E_{k}$ stores the corresponding eigenvectors as its column.

Inspired by the classical ICA formulation, an extra rotation $W^{T}$ applied to (7), the formulation (7),(8) can be rewritten as:

$$
\begin{gathered}
\mathrm{K}=\left(E D^{\frac{1}{2}} W\right)\left(W^{T} D^{\frac{1}{2}} E^{T}\right) \\
\widehat{V(p)}=\left(1_{n}^{T} E D^{\frac{1}{2}} W_{k}\right)^{2}
\end{gathered}
$$

The $W$ is an orthonormal linear transformation, i.e., $W W^{T}=I$. so that the covariance matrix does not change, but the modification allows us to maximize the "information potential" with respect to the number of retained components. The Optimized ECA algorithm is proposed to seek for optimally entropic data descriptors which may benefit classification.

The procedure of the Optimized ECA is: of S:

1. Calculate the covariance matrix of the input data set $S=\left[s_{1}, s_{2}, \ldots, s_{n}\right], \bar{s}$ denotes the mean value

$$
C_{e c a}=\frac{1}{n} \sum_{i=1}^{n}\left(\bar{s}-s_{i}\right)\left(\bar{s}-s_{i}\right)^{T}
$$


2. Eigen-decompose the $C_{e c a}$ :

$$
C_{e c a}=\left(E D^{\frac{1}{2}}\right)\left(D^{\frac{1}{2}} E^{T}\right)
$$

3.Applying the extra rotation matrix $\mathrm{W}$ to the covariance matrix $C_{\text {eca }}$ :

$$
C_{e c a}=\left(E D^{\frac{1}{2}} W\right)\left(W^{T} D^{\frac{1}{2}} E^{T}\right)
$$

4.Initialize the parameters $W, \tau$, the function $L(w)=\left(1_{n}^{T} E D^{\frac{1}{2}} w_{k}\right)^{2}$, where $w_{k}$ is the column vector and $w_{k} \in W,\left\|w_{k}\right\|_{2}=1$.

for $t$ iterations do

$$
\begin{array}{ll}
\text { 1. } & \mathrm{dJ}=\frac{\partial_{l(w)}}{\partial \mathrm{w}}=2\left(1_{n}^{T} E D^{\frac{1}{2}} w_{k}\right)\left(1_{n}^{T} E D^{\frac{1}{2}}\right) \\
\text { 2. } & w_{k}(n+1)=w_{k}(n)+\mathrm{T} * d J \\
\text { 3. } & E=E D^{\frac{1}{2}} w_{k}(n+1)
\end{array}
$$

end for

5. Making $B=E D^{\frac{1}{2}} W, v=\frac{1}{n^{2}} 1_{n}^{T} K 1_{n}=\sum_{j=1}^{N_{c}} \lambda_{j}^{\frac{1}{2}} 1_{n}^{T} e_{j}$, sort the $\mathrm{B}$ and $\mathrm{W}$ according to $v$.

In order to solve the OECA optimization problem, a gradient-ascend algorithm is used:

$$
w_{k}(n+1)=w_{k}(n)+\tau * \frac{\partial L}{\partial w_{k}(n)}
$$

Where is the step size, the gradient is

$$
\frac{\partial L}{\partial w_{k}(n)}=2\left(1_{n}^{T} E D^{\frac{1}{2}} w_{k}\right)\left(1_{n}^{T} E D^{\frac{1}{2}}\right)
$$

\section{Experiment and Result}

\subsection{The Proposed Method}

The proposed feature extraction method are combined with 2DGabor wavelet transformation and the Optimized ECA algorithm. 2DGabor algorithm is first applied to extract the 2DGabor feature, then the extracted feature is handled in 5 different ways, they are described as follow:

1. the 2DGabor feature $G_{u, v}$ is normalized:

$$
G_{u, v}(x, y)=\frac{\mathrm{G}(x, y)-\operatorname{mean}\left(G_{u, v}(x, y)\right)}{\operatorname{std}\left(G_{u, v}(x, y)\right)}
$$

We can get 40 2DGabor features, after the normalization, the extracted features are concentrated as a feature vector $\left\{\mathrm{A}: \mathrm{A}=G_{0,1}, G_{0,2}, \ldots, G_{4,7}\right\}$

2. the 2DGabor feature $G_{u, v}$ are fused according to the average weighting method in the eight directions of the five scales:

the results lists:

$$
\sum_{u=0}^{4} G_{u, v}(x, y)=\frac{1}{8} * \sum_{v=0}^{7} G_{u, v}(x, y)
$$

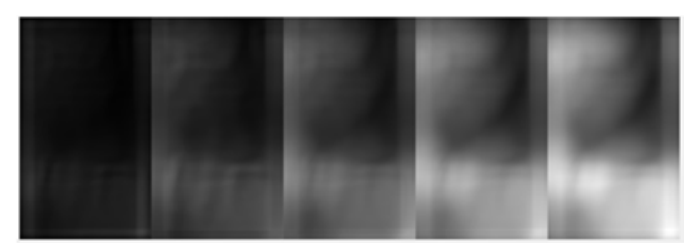

Figure 2. 2DGabor feature fusion in five scales

3. the 2DGabor feature $G_{u, v}$ are fused according to the average weighting method in the five scales directions of the eight directions:

$$
\sum_{v=0}^{7} G_{u, v}(x, y)=\frac{1}{5} * \sum_{u=0}^{4} G_{u, v}(x, y)
$$

The result lists: 


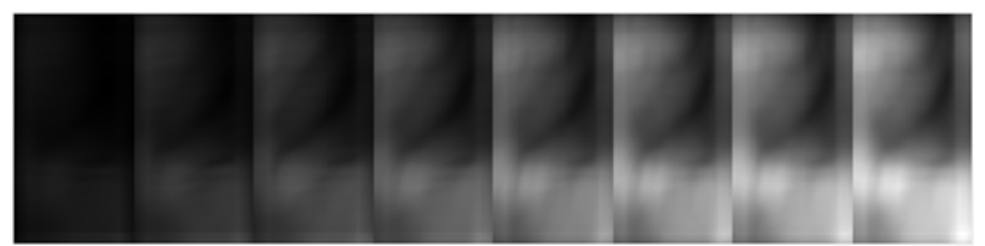

Figure 3. 2DGabor feature fusion in eight directions

4. the 2DGabor feature $G_{u, v}$ are fused according to the average weighting method in the eight directions of the five scales after the Local Binary Pattern algorithm, use $G B_{u, v}(x, y)$ instead of $G_{u, v}(\mathrm{x}, \mathrm{y})$ :

The result lists:

$$
\sum_{u=0}^{4} G B_{u, v}(x, y)=\frac{1}{8} * \sum_{v=0}^{7} G B_{u, v}(x, y)
$$

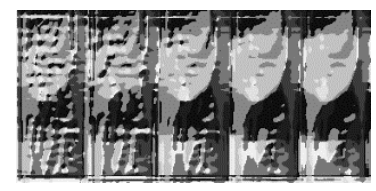

Figure 4. 2DGabor feature coded by LBP fusion in five scales

5. the 2DGabor feature $G_{u, v}$ are fused according to the average weighting method in the five scales of the eight directions after the Local Binary Pattern algorithm, use $G B_{u, v}(x, y)$ instead of $G_{u, v}(\mathrm{x}, \mathrm{y})$ :

The result lists:

$$
\sum_{v=0}^{7} G B_{u, v}(x, y)=\frac{1}{5} * \sum_{u=0}^{4} G B_{u, v}(x, y)
$$

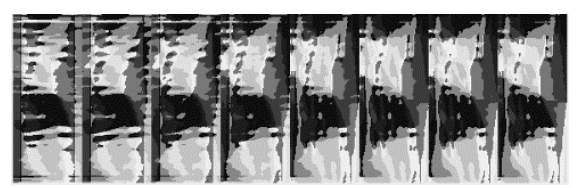

Figure 5. 2DGabor feature coded by LBP fusion in eight directions

the five 2DGabor features are all combined with the Optimized ECA algorithm to reduce the dimension then use the Nearest Neighbor classifier for classification.

\subsection{Finger Vein Database}

The finger vein database is from the key lab of intelligent signal and image processing of Tianjin Civil Aviation University. The database contains 64 persons' samples, each one has 15 samples, after the preprocessing, the samples are normalized into $180 * 75$ pixels. The following figure shows some of the sample images from different people,

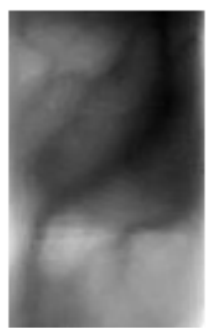

\subsection{Experiment Result}
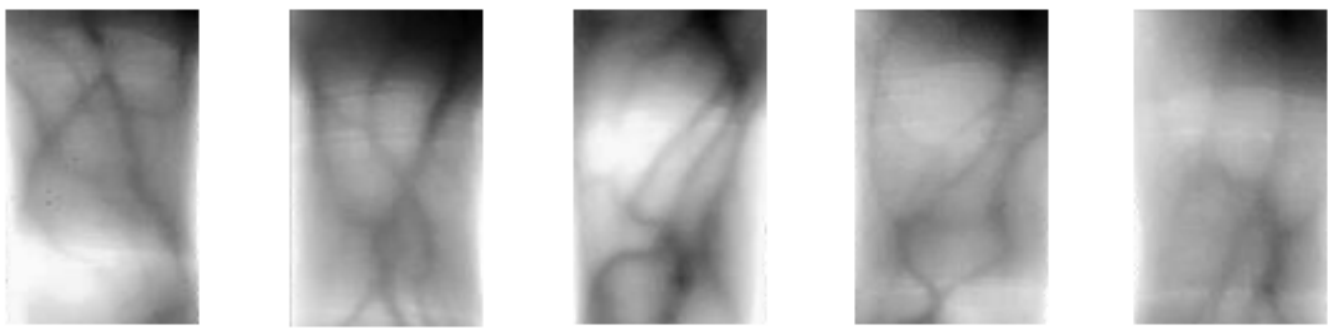

Figure 6. Finger vein samples from different people

Table 1 shows the result of finger vein recognition based on the database, 
Table 1. The recognition time and rate of different methods

\begin{tabular}{ccc}
\hline Experiment Results & $\begin{array}{c}\text { Mean recognition } \\
\text { rate } \\
\text { (percentage) }\end{array}$ & $\begin{array}{c}\text { Mean recognition } \\
\text { time(ms) }\end{array}$ \\
\hline Feature category & 88.12 & 26.098 \\
PCA & 89.11 & 22.891 \\
OECA & 90.81 & 48.545 \\
$\begin{array}{c}\text { 2DGabor+feature fusion } \\
\text { 1+OECA }\end{array}$ & 99.21 & 1836.673 \\
2DGabor+feature fusion & 98.91 & 150.420 \\
2+OECA & 98.28 & 152.233 \\
2DGabor+ feature fusion & 99.06 & 180.837 \\
3+OECA & 99.06 & 186,823 \\
2DGabor+ feature fusion & 4+OECA &
\end{tabular}

The experiment data prove the proposed OECA algorithm and the 2DGabor combined with OECA algorithm is efficient in finger vein recognition. The feature fusion rule 1 cost much recognition time although its recognition rate is higher, The feature fusion rule 2,3 cost less time and the recognition rates are acceptable. The feature fusion rule 4,5 achieve higher recognition rate. The feature fusion rule 2-4 meet the requirement of real-time identification of the venous system.

\section{Conclusion}

Finger vein recognition is popular to researchers for its stability and safety, and lower computing complexity. In this paper, we introduce the research and development of finger vein recognition, then proposed the Optimized ECA algorithm based on the optimized KECA algorithm. The Optimized ECA is an extension of ECA, which get much expressive features, 2DGabor wavelet transform is much efficient in ridge feature analysis so we propose a method combined the 2DGabor with Optimized ECA, then use this method in finger vein recognition. Experiment results confirm the recognition rate of feature fusion less than $100 \%$, but it is an efficient method.

\section{References}

[1]. Kono, Miyuki, Hironori Ueki, and Shin-ichiro Umemura. "A new method for the identification of individuals by using of vein pattern matching of a finger." Proceedings of the 5th symposium on pattern measurement, Yamaguchi, Japan. 2000.

[2]. US Patent no. 7,526,111 "Personal Identification Device and Method"

[3]. Yin Ying- Long, et al. "Study of finger vein recognition”.Journal of Data Acquisition and Processing, 2015, 30 (5): 933-939.

[4]. Wang Yi-Ding, et al. "Study on identity recognition using hand dorsal vein ” .Journal of HighTech Communication, 2014, 24 (6): 632-642.

[5]. Yu, Cheng-Bo, et al. "Finger-vein image recognition combining modified hausdorff distance with minutiae feature matching." Interdisciplinary Sciences: Computational Life Sciences 1.4 (2009): 280-289.

[6]. Qin, Hua-feng, et al. "Finger-vein verification based on multi-features fusion." Sensors 13.11 (2013): 15048-15067.

[7]. Liu, T., et al. "An algorithm for finger-vein segmentation based on modified repeated line tracking." The Imaging Science Journal 61.6 (2013): 491-502.

[8]. Qin, Huafeng, Lan Qin, and Chengbo Yu. "Region growth-based feature extraction method for finger-vein recognition." Optical Engineering 50.5 (2011): 057208-057208. 
[9]. Miura, Naoto, Akio Nagasaka, and Takafumi Miyatake. "Extraction of finger-vein patterns using maximum curvature points in image profiles." IEICE TRANSACTIONS on Information and Systems 90.8 (2007): 1185-1194.

[10]. Rosdi, Bakhtiar Affendi, Chai Wuh Shing, and Shahrel Azmin Suandi. "Finger vein recognition using local line binary pattern." Sensors 11.12 (2011): 11357-11371.

[11]. Yang, Jinfeng, Yihua Shi, and Jinli Yang. "Finger-vein recognition based on a bank of Gabor filters." Computer Vision-ACCV 2009 (2010): 374-383.

[12]. Wu, Jian-Da, and Chiung-Tsiung Liu. "Finger-vein pattern identification using principal component analysis and the neural network technique." Expert Systems with Applications 38.5 (2011): 5423-5427.

[13]. Jia, Xue-Mei, et al. "A New Method Combining KECA-LDA with ELM for Classification of Chinese Liquors Using Electronic Nose." IEEE Sensors Journal 16.22 (2016): 8010-8017.

[14]. El Madany, et al. "Human action recognition using temporal hierarchical pyramid of depth motion map and keca." Multimedia Signal Processing (MMSP), 2015 IEEE 17th International Workshop on. IEEE, 2015.

[15]. Jenssen, Robert. "Kernel entropy component analysis." IEEE transactions on pattern analysis and machine intelligence 32.5 (2010): 847-860.

[16]. Izquierdo-Verdiguier, Emma, et al. "Optimized kernel entropy components." IEEE transactions on neural networks and learning systems (2016).

[17]. Principe, Jose C. Information theoretic learning: Renyi's entropy and kernel perspectives. Springer Science \& Business Media, 2010. 